\section{Evolution of the orang-utan}

SMITH AND PILBEAM ${ }^{1}$ have argued that "the available evidence... justifies the concept of a Pliocene orang-utan ancestor at least as terrestrial as the modern chimpanzee". It is, however, questionable whether they have established their thesis as equally, or more, plausible than the one they wish to replace (that the ancestral lineage of orang-utans was arboreal).

The authors have used both palaeontological and neontological records, but their arguments are unconvincing. First, there are no unequivocal data on the body sizes of Pleistocene orang-utans so that inferences on arboreality or terrestriality are tenuous. Although Smith and Pilbeam acknowledge the difficulty of drawing conclusions about body size from tooth size alone when considering subfossil orang-utan populations, they conclude that mid-Pleistocene mainland forms were larger-bodied and hence less arboreal than the extant form. The basis for such a conclusion is not evident. Second, plausibility alone does not constitute empirical (or theoretical) support for a thesis. Smith and Pilbeam suggest that it is plausible to view the large body size and marked sexual dimorphism of living orang-utans as remnants of a more terrestrial pattern. This is a possibility but they offer no direct support. Thus there is no reason to consider this alternative of greater importance than any other (sexual selection, niche separation, and such ) that may have an influence on whether or not animals are terrestrial. Third, $\mathrm{Kay}^{2}$ has shown that (1) there is no relationship between tooth enamel thickness and arboreality or terrestriality, and (2) thick enamel, as found in Pongo, seems to be associated with the consumption of hard fruits, nuts and seeds. This weakens arguments by Smith and Pilbeam that ancestral orang-utans may not have been arboreal frugivores.

If there are data that do not support a particular hypothesis, then the inconsistency should be explained. The postcranial anatomy of living orang-utans is the least equivocal source of data on possible "remnants... of a more terrestrial pattern". Morphological studies of orang-utan cheiridia ${ }^{3}$, wrist $^{4}$, and hip and thigh $^{3}$ have all revealed marked specializations consistent with arboreal progression in a large-bodied primate. No features suggesting adaptations for terrestrial locomotion, present or past, have been reported. Smith and Pilbeam have not accorded these data the attention they deserve, nor explained why extant orangutans should not display evidence of terrestriality in an ancestral form. It is unclear, for example, whether the authors postulate a stage of terrestriality so brief that terrestrial adaptations had not time to evolve, or, if arguing for a longer period of terrestriality, whether the return to arboreal progression was to have been accompanied by an exact reversal of evolutionary changes.

Despite the available fossil evidence suggesting that "typical Neogene hominoids (including our hypothetical Pliocene orang-utan) were probably ... woodland creatures", one cannot yet eliminate the possibility that this is a simple artefact of the still very limited fossil record. Similarly, there is no reason to expect that all fossils displaying an affinity to extant forms represent populations directly ancestral to living species. It may be worthwhile to consider the possibility, for example, that the midPleistocene orang-utans represented by fossil teeth were a related, but not ancestral, species. Until the competing hypotheses of arboreality or terrestriality can be tested by unequivocal data from the fossil record, they must be considered viable alternatives.

I thank H. McHenry, P. Rodman and L. Scott for reading an earlier version of this manuscript.

\section{Department of Anthropology, \\ University of California, Davis, California 95616}

1. Smith, R. J. \& Pilbeam, D. R. Nature 284, 447-448 (1980). 2. Kay, R. Am. J. phys. Anthrop. 52, 243 (1980).

3. Tuttle, R. H. J. Morph. 128, 309-364 (1969); The Chimpanzee 2, 167-253 (1970); Gibbon Siamang 1, 136-206 (1972).

4. Lewis, O. J. Am. J. phys. Anthrop. 30, 251-268 (1969); in Primate Locomotion (ed. Jenkins, F. A.) 143-169 (Academic, New York, 1974).

5. Sigmon, B. A. J. Hum. Evol. 3, 161-185 (1974).

SMITH AND PILbEAM REPLY - We do not know whether Miocene ancestors of the orang-utan were arboreal or terrestrial, and evidence marshalled in favour of either hypothesis is tenuous at best. Nevertheless, it seems to us that hypotheses for hominoid evolution generally have assumed with confidence that the orang lineage has always been arboreal. In offering evidence for the possibility of a terrestrial ancestor, our purpose was also to lead others to reevaluate the supposed evidence for arboreality. We completely agree with Temerin's conclusion that arboreality and terrestriality "must be considered viable alternatives".

\section{RICHARD J. SMITH}

Department of Orthodontics,

Dental School,

University of Maryland,

Baltimore, Maryland 21201

DAVID R. PILBEAM

Department of Anthropology,

Yale University,

New Haven, Conneticut 06520

\section{Noradrenergic transmission}

I WISH to raise two points concerning the discussion and conclusion in a recent report by Hirst and Nield ${ }^{1}$.

First, evidence quoted to illustrate that a portion of sympathetic postganglionic transmission is resistant to adrenoreceptor antagonists was derived from work on rodent vasa deferentia: the authors had concluded that in these tissues a component of the motor transmission was 'non-adrenergic' (refs. 2-5). From the reported results ${ }^{1}$, however, noradrenergic transmission resistant to $\alpha$-adrenoreceptor antagonists is postulated. This conclusion may be justified in the case of the arteriolar preparation under study and, if so, constitutes an important observation. However, if this is offered as an explanation for the 'non-adrenergic' component of transmission in vas deferens, it should be noted that all previous reports quoted as having supported this latter concept, did so not only on the evidence of resistance to antagonists but also because the response persisted after depletion of noradrenaline by reserpine, for example, to less than $1 \%$ of control levels ${ }^{6}$. Furthermore, responses persisted even after chemical sympathectomy when virtually all adrenergic nerve terminals had been removed ${ }^{7}$. In contrast, the 'conventional', adrenergic, $\alpha$-blockersusceptible component was completely absent following reserpine or chemical sympathectomy $y^{2,7}$. If allowance for this adrenergic component in the control is made, the 'non-adrenergic' response is not reduced at all following the latter treatments $^{2,8}$. There is, therefore, no evidence to connect this response in vas deferens with noradrenaline or noradrenergic nerves. To associate this with a discussion of the postulated mechanism in arteriolar muscle can only attract irrelevant criticism of the latter. It is also possible that the intracellular excitatory junctional potential and the 'nonadrenergic' contraction of the muscle layers reflect different cellular processes.

Secondly, the conclusion that there are 'two populations of excitatory receptors for noradrenaline in arteriolar smooth muscle' could be confused with the other recent evidence for two distinct types of excitatory $\alpha$-adrenoreceptor on vascular and non-vascular smooth muscle ${ }^{9,10}$; in this case, each response was blocked by the appropriate antagonist ${ }^{9-11}$. Furthermore, in contrast to the newly reported observation on arteriolar muscle ${ }^{1}$, the pressor response to sympathetic nerve stimulation was even more sensitive to blockade by the $\alpha_{1}$-adrenoreceptor antagonist, prazosin, than was the response to exogenous noradrenaline ${ }^{11,13}$. The 'phentolamine-resistant' effect of 